\title{
Research Highlights Reversing Cocaine-Induced Adaptations and Reducing Relapse: An Opportunity for Repurposing Riluzole
}

\author{
Joseph J Ziminski' and Eisuke Koya*,I \\ 'Sussex Neuroscience, School of Psychology, University of Sussex, Falmer, UK
}

Neuropsychopharmacology (2018) 43, I 197-I 198; doi:I0.1038/npp.2017.300; published online 17 January 2018

High rates of relapse to drug taking are often triggered by exposure to drug-associated cues or drugs of abuse, and they present a major obstacle for the successful treatment of drug addiction (O'Brien, 2005). Despite years of intensive research efforts to date, there is a paucity of effective medications to treat relapse. A core principle behind discovering potentially effective drugs to reduce relapse has been to identify pharmacological compounds that reverse drug-induced changes in the brain, in particular those areas implicated in motivation and reward, such as the medial prefrontal cortex (mPFC) and nucleus accumbens. A plethora of pre-clinical studies from the last 10-15 years have revealed that repeated cocaine exposure produces abnormalities in intrinsic neuronal excitability, glutamatergic synaptic plasticity, and glutamate uptake, in the mPFC and accumbens (Kalivas and Kalivas, 2016; Kourrich et al, 2015). Thus, it would be highly desirable for an anti-relapse compound to renormalize these types of widespread drug-induced changes. Moreover, a compound with a known safety record that has already passed clinical trials and existing on the market would greatly enhance its desirability.

In this issue of Neuropsychopharmacology, SepulvedaOrengo et al, 2017 investigated whether Riluzole, a compound that is currently in use for treating amyotrophic lateral sclerosis (Traynor et al, 2006), could renormalize these types of adaptations and prevent the reinstatement of cue-induced and cocaine-primed cocaine-seeking. The authors took advantage of this compound because of its multi-target directed action to (1) block action potential (AP) initiation via inhibiting voltage-gated $\mathrm{Na}^{+}$channels (Cheah et al, 2010); (2) increase expression and activity of glutamate transporters (Brothers et al, 2013); and (3) inhibit synaptic glutamate release via $\mathrm{Ca}^{+2}$ channel inhibition (Cheah et al, 2010). Initially, the authors repeatedly administered Riluzole during extinction learning (before each extinction session) and before the reinstatement test, which attenuated reinstatement of cue- and cocaine-primed cocaine seeking.

\footnotetext{
*Correspondence: Dr E Koya, Sussex Neuroscience, School of Psychology, University of Sussex, CRPC 5.14, Falmer BNI9QG, UK, Tel: +44 1273877 776, E-mail: e.koya@sussex.ac.uk
}

This effect was not due to any locomotor inactivating effects of the compound, as repeated Riluzole administration did not attenuate the reinstatement of cue-induced sucrose seeking nor general locomotor activity.

Next, using brain slice electrophysiology, SepulvedaOrengo et al, 2017 examined whether Riluzole would reverse the intrinsic excitability adaptations in pyramidal cells from the prelimbic (PL) and infralimbic (IL) regions of the mPFC following extinction from cocaine self-administration. Such intrinsic excitability adaptations modulate the ability of a neuron to elicit an AP and thus communicate with other neurons. The PL and IL areas have distinct roles in cocaine seeking, in which the PL functions to drive responding while the IL mediates response inhibition (Gourley and Taylor, 2016; Peters et al, 2008). The authors found that PL and IL neuronal excitability was enhanced and attenuated, respectively, at the level of firing capacity following electrical current injections. Underlying these changes in intrinsic excitability were alterations in the fast after hyperpolarization component of the AP, which contributes to the repolarization phase of the AP. Furthermore, decreased expression of the glutamate transporter 'GLT-1' in the nucleus accumbens was also observed. This molecule critically controls extracellular glutamate levels by removing glutamate, and renormalizing cocaine-induced decreases of this molecule has been shown to decrease cocaine-seeking (Kalivas and Kalivas, 2016). Interestingly, Riluzole reversed both the excitability adaptations in the PL and IL, and the decreases in accumbens GLT-1 expression. Taken together, these results suggest that Riluzole exerts its efficacy by restoring these cocaine-induced changes in corticostriatal areas.

These promising findings demonstrate Riluzole as a potentially valuable therapeutic tool to manage cocaine relapse. However, key questions remain regarding its mode of action. First, the authors administered Riluzole both during extinction learning and before the reinstatement test. It will next be important to ascertain whether chronic administration of Riluzole across extinction learning or acute exposure prior to the relapse test was responsible for the renormalization of cocaine-induced adaptations and attenuation of reinstatement. If Riluzole's action is mediated by repeated interactions with 
neuronal activity during cocaine extinction, then this compound might serve as a complementary pharmacotherapy during 'cue exposure therapy' that utilizes principles of extinction learning. Alternatively, if its actions are acute, then this property might be exploited to rapidly interfere with motivational states that trigger relapse, such as drug cravings (O'Brien, 2005), and help sustain recovery from drug addiction. Moreover, will repeated Riluzole treatment remain efficacious in the absence of extinction learning? This crucial question is particularly relevant as addicts may relapse following exposure to drug cues following prolonged abstinence in which they may not be exposed to any drug cues (eg, during a prison sentence and rehabilitation).

Finally, exposure to chronic cocaine and other drugs of abuse modulate glutamate transmission in the nucleus accumbens (Kalivas and Kalivas, 2016). Thus, the promising results of Sepulveda-Orengo et al, 2017 raise the question of whether Riluzole can prevent the development of cocaineinduced excitatory synaptic potentiation in accumbens medium spiny neurons, or attenuate reinstatement of drugseeking for other drugs of abuse (eg, alcohol, nicotine, and opiates). If Riluzole prevents this synaptic potentiation and broadly acts on various drug-seeking behaviours, then such evidence can be used to provide an even more convincing case for its use as an anti-relapse medication.

In summary, this study elegantly demonstrates how Riluzole reverses cocaine-induced changes in intrinsic excitability and GLT-1 levels in corticostriatal brain areas, and dampen cocaine-seeking. Although a previous clinical study could not demonstrate Riluzole's efficacy in the treatment of cocaine dependence, this small-scale study should be taken with caution since the authors did not examine patient compliance (Ciraulo et al, 2005). Thus, at the present stage, further comprehensive clinical studies that examine Riluzole's potential in relapse management are still required, and it will take many years until this compound may be approved for such purposes. However, Riluzole is already available on the pharmaceutical market. Therefore, unlike drugs undergoing clinical trials, Riluzole has the advantage of being potentially 'repurposed' as an anti-relapse compound and the critical findings of Sepulveda-Orengo et al, 2017 may represent a crucial initial step in this process.

\section{FUNDING AND DISCLOSURE}

The authors declare no conflict of interest.

\section{ACKNOWLEDGMENTS}

Research of the authors is supported by BBSRC grant BB/ M009017/1, MRC Discovery Award, and the Sussex Strategic Development Funds.

\section{REFERENCES}

Brothers HM, Bardou I, Hopp SC, Kaercher RM, Corona AW, Fenn AM et al (2013). Riluzole partially rescues age-associated, but not LPS-induced, loss of glutamate transporters and spatial memory. J Neuroimmune Pharmacol 8: 1098-1105.

Cheah BC, Vucic S, Krishnan AV, Kiernan MC (2010). Riluzole, neuroprotection and amyotrophic lateral sclerosis. Curr Med Chem 17: 1942-2199.

Ciraulo DA, Sarid-Segal O, Knapp CM, Ciraulo AM, LoCastro J, Bloch DA et al (2005). Efficacy screening trials of paroxetine, pentoxifylline, riluzole, pramipexole and venlafaxine in cocaine dependence. Addict Abingdon Engl 100(Suppl 1): 12-22.

Gourley SL, Taylor JR (2016). Going and stopping: dichotomies in behavioral control by the prefrontal cortex. Nat Neurosci 19: 656-664.

Kalivas BC, Kalivas PW (2016). Corticostriatal circuitry in regulating diseases characterized by intrusive thinking. Dialogues Clin Neurosci 18: 65-76.

Kourrich S, Calu DJ, Bonci A (2015). Intrinsic plasticity: an emerging player in addiction. Nat Rev Neurosci 16: 173-184.

O'Brien CP (2005). Anticraving medications for relapse prevention: a possible new class of psychoactive medications. Am J Psychiatry 162: 1423-1431.

Peters J, LaLumiere RT, Kalivas PW (2008). Infralimbic prefrontal cortex is responsible for inhibiting cocaine seeking in extinguished rats. J Neurosci 28: 6046-6053.

Sepulveda-Orengo MT, Healey KL, Kim R, Auriemma AC, Rojas J, Woronoff $\mathrm{N}$ et al (2017). Riluzole impairs cocaine reinstatement and restores adaptations in intrinsic excitability and GLT-1 expression. Neuropsychopharmacology (this issue).

Traynor BJ, Bruijn L, Conwit R, Beal F, O’Neill G, Fagan SC et al (2006). Neuroprotective agents for clinical trials in ALS: a systematic assessment. Neurology 67: 20-27. 